\title{
Controle da Ferrugem do Cafeeiro com Base no Valor de Severidade
}

\author{
Clévio L. P. Garçon ${ }^{1}$, Laércio Zambolim¹, Eduardo S. G. Mizubuti ${ }^{1}$, Francisco X. R. do Vale ${ }^{1}$, Hélcio Costa ${ }^{2}$
}

'Departamento de Fitopatologia, Universidade Federal de Viçosa, CEP 36571-000, Viçosa, MG, fax: (031) 3899-2240,

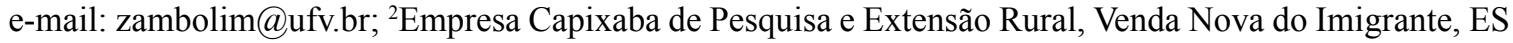

(Aceito para publicação em 28/07/2004)

Autor para correspondência: Laércio Zambolim

GARÇON, C.L.P., ZAMBOLIM, L., MIZUBUTI, E.S.G., VALE, F.X.R. \& COSTA, H. Controle da ferrugem do cafeeiro com base no valor de severidade. Fitopatologia Brasileira 29:486-491. 2004.

\section{RESUMO}

O presente trabalho teve por objetivo avaliar o controle da ferrugem do cafeeiro (Coffea arabica) baseando-se no valor de severidade, calculado em função das variáveis meteorológicas, molhamento foliar e temperatura média durante o período de molhamento. Foram escolhidas duas lavouras de café da cultivar Catuaí Vermelho, uma com alta carga pendente de frutos (101,5 sacas beneficiadas/ha), com seis anos de idade, localizada no município de Coimbra, a $680 \mathrm{~m}$ de altitude, na região da Zona da Mata de Minas Gerais; e a outra, com carga média de frutos (22,4 sacas beneficiadas/ha), com nove anos de idade, localizada no município de Carmo do Paranaíba, a $850 \mathrm{~m}$ de altitude, na região do Alto Paranaíba do Estado de Minas Gerais. Em Coimbra, quando o valor de severidade da ferrugem (VSF) foi igual a 30, foram feitas duas pulverizações com fungicida sistêmico, igualando-se as duas aplicações de fungicida sistêmico do calendário.
Em Carmo do Paranaíba, o VSF igual a 49 recomendou apenas uma única pulverização; todos os outros tratamentos demandaram duas aplicações de fungicidas sistêmicos. A incidência final de folhas doentes na colheita $(11,0 \%)$ para todos os tratamentos, não atingiu o nível de dano econômico ao cafeeiro. Não houve diferença na eficiência de controle dos tratamentos baseados no calendário (duas aplicações de fungicidas sistêmicos e quatro aplicações de fungicida cúprico), duas aplicações de fungicida sistêmico baseadas na incidência da doença $(10 \%)$ e o sistema baseado no VSF. Portanto, o sistema baseado no VSF foi tão eficiente quanto o calendário, para o controle da ferrugem do cafeeiro, porém com economia de uma pulverização, na lavoura com carga média de frutos (Carmo do Paranaíba).

Palavras-chave adicionais: Coffea arabica, controle integrado, fungicida.

ABSTRACT

\section{Coffee leaf rust control based on rust severity values}

This trial evaluated coffee (Coffea arabica) leaf rust control based on rust severity values (RSV) according to climatic variables such as leaf wetness and air temperature during leaf wetness period. Two coffee growing areas were chosen, both in the state of Minas Gerais: a six-year-old coffe plantation in Coimbra in the Zona da Mata at $680 \mathrm{~m}$ of altitude, and a nine-year-old coffee plantation in Carmo do Paranaíba in the Alto Paranaíba at $850 \mathrm{~m}$ of altitude. In Coimbra, a potentially high yielding coffee plantation, the treatment
\end{abstract}

based on RSV of 30 was similar to the calendar treatment (two sprays of systemic fungicides). In Carmo do Paranaíba, a potentially medium yielding coffe plantation, the treatment based on RSV of 49 recommended one application of systemic fungicide. The treatment based on the calendar preconized two applications for the systemic and four applications of the protective fungicide. In conclusion, the system based on RSV was as good as the calendar treatment for high berry yield coffee plants; for the medium berry yield coffee plants the system reduced at least one fungicide application.

\section{INTRODUÇÃo}

A ferrugem do cafeeiro (Coffea arabica L.) é causada por Hemileia vastatrix Berk. et Br., espécie descrita por Berkeley, para enquadrar a ferrugem encontrada em café, em 1869, no Sri Lanka (Zambolimet al., 1997).

Os danos causados pela ferrugem são, principalmente, indiretos, pela indução de desfolha por ocasião da colheita. A queda precoce das folhas resulta em menor vingamento da florada, menor vingamento dos chumbinhos e também seca dos ramos plagiotrópicos, comprometendo, em alguns casos em mais de 50\%, a produção do cafeeiro (Gree, 1993; Zambolim et al., 1997).

Considerações sobre o uso de fungicidas na agricultura, como oneração do custo de produção, degradação dos recursos naturais, problemas de intoxicação de aplicadores de defensivos agrícolas, aumento dos riscos da presença de resíduos nos produtos colhidos, assim como, surgimento de raças do fungo resistentes têm levado a uma procura crescente por práticas de manejo de doenças mais racionais e por fungicidas de menores custos e toxicidade (Zambolim \& Vale, 1999).

Fazendo-se o monitoramento das variáveis meteorológicas é possível identificar períodos de condições favoráveis às doenças, permitindo o estabelecimento dos momentos mais apropriados a aplicações de fungicidas. Desta maneira, podese obter informações sobre quando as pulverizações devem ser iniciadas e o intervalo que elas devem ser feitas ou, ainda, se devem ser feitas com a mesma freqüência em todas as épocas do ano (Campbell \& Madden, 1990).

As previsões de epidemias causadas por patógenos dispersos pelo ar começaram em 1926, trazendo grandes 
Controle da ferrugem do cafeeiro com base no valor...

perspectivas para a otimização do controle de doenças de plantas. Os primeiros trabalhos desenvolvidos visaram doenças como a requeima da batata (Solanum tuberosum L.), o míldio da videira (Vitis spp.) e a sarna da macieira (Malus domestica Borkh.) (Rotem, 1994).

No Brasil, trabalhos visando entender a influência do clima e do hospedeiro sobre a ferrugem do cafeeiro, vêm sendo realizados no Brasil desde há muito tempo (Alfonsiet al., 1974; Vale et al., 2000; Zambolim et al., 2002). Referidos autores estudaram a influência das variáveis climáticas sobre o período de incubação, número médio de pústulas por folha da ferrugem do cafeeiro, e área foliar das plantas.

$\mathrm{Na}$ década de 80, foi proposto por Kushalappa et al. (1983) um modelo de previsão da ferrugem do cafeeiro baseado na razão da sobrevivência líquida para o processo monocíclico adotando variáveis importantes dos três vértices do triângulo da doença: o patógeno, o ambiente e o hospedeiro, para explicar a evolução da doença.

Diante desses fatos, avaliou-se neste trabalho o valor de severidade da ferrugem (VSF) do cafeeiro, como critério para seu controle químico, por meio da aplicação de fungicidas sistêmicos. Portanto, o objetivo do trabalho foi desenvolver um sistema simples que permitisse prever de maneira confiável o desenvolvimento da doença no campo e, assim, determinar o momento propício para iniciar seu controle químico por meio de pulverizações com fungicida sistêmico, bem como o intervalo entre as aplicações.

\section{MATERIALEMÉTODOS}

Para a condução do experimento foram escolhidas duas lavouras do cultivar Catuaí Vermelho. Uma com alta carga pendente de frutos (101,5 sacas beneficiadas/ha), localizada no Sítio Boa Vista no município de Coimbra na Zona da Mata Mineira, a $680 \mathrm{~m}$ de altitude; e a outra com média carga pendente (22,4 sacas beneficiadas/ha), localizada no município de Carmo do Paranaíba na região do Alto Paranaíba no Estado de Minas Gerais a $850 \mathrm{~m}$ de altitude.

As variáveis climáticas empregadas para cálculo do VSF foram o molhamento foliar diário e temperatura média durante o período de molhamento foliar, obtidas em uma estação meteorológica colocada no meio da área experimental munida de sensor de molhamento foliar, temperatura, umidade relativa e precipitação pluvial.

Com os dados diários dessas variáveis meteorológicas calculou-se o VSF, construindo-se uma matriz dos VSF, semelhante à idealizada por Wallin (1962), modificada para a ferrugem do cafeeiro (Tabela 1).

O delineamento experimental foi blocos ao acaso com 11 tratamentos e quatro repetições, tendo cada parcela dez plantas úteis e duas plantas como bordadura. O teste de agrupamento Scott-Knott foi empregado para separar grupos de tratamentos distintos quanto a AACPD com base na incidência de folhas de café com ferrugem.

As avaliações do progresso da doença no campo foram feitas mensalmente, determinando-se a incidência da ferrugem (porcentagem de folhas com uredosporos) por parcela. As amostragens foram realizadas retirando-se dez folhas por planta, nas posições dos pontos cardeais, no terceiro ou quarto par de folhas completamente desenvolvidas, coletadas ao acaso, nos ramos do terço inferior das plantas. Na avaliação optou-se pela incidência da ferrugem pelo fato de correlacionar com a severidade da doença (Maffia et al., 1988).

Descrição dos tratamentos, para lavoura com alta carga pendente de frutos: 1 - Testemunha: sem pulverização; 2 Oxicloreto de Cobre 500 PM: 3,0 kg/ha em Dez/99, Jan/00, Fev/ 00 e Mar/00 (calendário); 3 - Epoxiconazole 250 SC: 0,6 1/ha em Jan/00 e 0,4 1/ha em Mar/00 (calendário); 4 - Tetraconazole 100 CE: 0,5 1/ha em Jan/00 e Mar/00 (calendário); 5 - Hexaconazole 100 SC: 0,5 1/ha em Jan/00 e Mar/00 (calendário); 6 Epoxiconazole 250 SC: 0,6 1/ha quando atingir o índice de 10\% de incidência, e 0,4 1/ha 60 dias após primeira; 7 - Hexaconazole 100 SC: $0,51 /$ ha quando atingir o índice de $10 \%$ de incidência, e 60 dias após primeira; 8 - Hexaconazole $100 \mathrm{SC}$ : 0,5 l/ha quando atingir VSF 29; 9 - Hexaconazole 100 SC: 0,5 l/ha quando atingir VSF 34; 10 - Hexaconazole $100 \mathrm{SC}: 0,5 \mathrm{l} / \mathrm{ha}$ quando atingir VSF 39; 11 - Hexaconazole $100 \mathrm{SC}$ : 0,5 1/ha quando atingir VSF 44.

$\mathrm{Na}$ lavoura com média carga pendente de frutos (Carmo do Paranaíba), foram alterados apenas os tratamentos baseados no VSF do tratamento 8 para 49; do tratamento 9 para 59; do tratamento 10 para 69 e do tratamento 11 para 79 . A alteração do VSF deveu-se ao fato de que, em plantas com média a baixa carga de frutos de café, a intensidade da ferrugem é baixa e em muitos casos não atinge nível de controle (Miguel et al., 1977; Zambolim et al., 1992).

\section{RESULTADOE DISCUSSÃO}

Controle baseado no SFL em lavoura com alta carga pendente de frutos.

As epidemias de ferrugem iniciaram-se no município de Coimbra em dezembro de 1999 e alcançaram os valores máximos em maio - junho de 2000 (Figura 1), chegando a $84,5 \%$ de incidência no tratamento testemunha.

TABELA 1 - Matriz para cálculo dos valores de severidade da ferrugem (VSF) do cafeeiro (Coffea arabica), com base no período de molhamento foliar e na temperatura média do período

\begin{tabular}{|c|c|c|c|c|c|c|c|}
\hline \multirow{2}{*}{$\begin{array}{l}\text { Molhamento } \\
\text { Foliar (h/diárias) }\end{array}$} & \multicolumn{7}{|c|}{ Temperatura $\left({ }^{\circ} \mathrm{C}\right)$} \\
\hline & $<16$ & $16-18$ & $19-20$ & 21-24 & $25-26$ & $27-29$ & 30 \\
\hline 0 & $0^{*}$ & 0 & 0 & 0 & 0 & 0 & 0 \\
\hline $0<\mathrm{h} \leq 8$ & 0 & 0 & 1 & 2 & 1 & 0 & 0 \\
\hline $8<\mathrm{h} \leq 17$ & 0 & 1 & 2 & 3 & 2 & 1 & 0 \\
\hline $17<\mathrm{h} \leq 24$ & 0 & 2 & 3 & 4 & 3 & 2 & 0 \\
\hline $\mathrm{h}=24^{* *}$ & 0 & 0 & 1 & 2 & 1 & 0 & 0 \\
\hline
\end{tabular}

* Valor de severidade da ferrugem (VSF) diário

** Molhamento foliar diário de $24 \mathrm{~h}$, porém este dentro de um período de molhamento de mais de $48 \mathrm{~h}$ sem interrupção.

OBS.: Se dentro de 30 dias não houver acumulado mais de 5 VSF, desconsideram os VSF's acumulados até o momento. 


\section{C.L.P. Garçon et al.}

A fase log da curva de progresso da doença para o tratamento testemunha, foi de janeiro de 2000 a abril de 2000; porém, as infecções que surgiram a partir de abril de 2000, foram decorrentes de condições ocorridas anteriormente. Duas são as explicações para o fato: a primeira, é a influência do clima no progresso da ferrugem do cafeeiro, onde o número de horas favoráveis à infeção (NHFI) do cafeeiro por H. vastatrix é decorrente da condição de molhamento foliar com temperatura entre $20-25^{\circ} \mathrm{C}$ (Vale et al., 2000). A partir de abril de 2000, as condições de temperatura média durante o período de molhamento foliar foram desfavoráveis à ocorrência de novas infecções, uma vez que $64,81 \%$ das horas de molhamento foliar deste período apresentaram temperatura inferiores a $16^{\circ} \mathrm{C}$, o

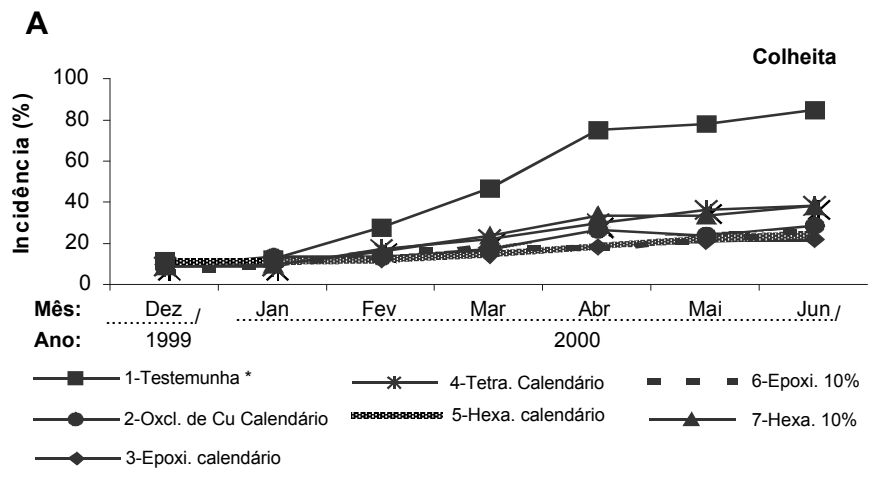

B

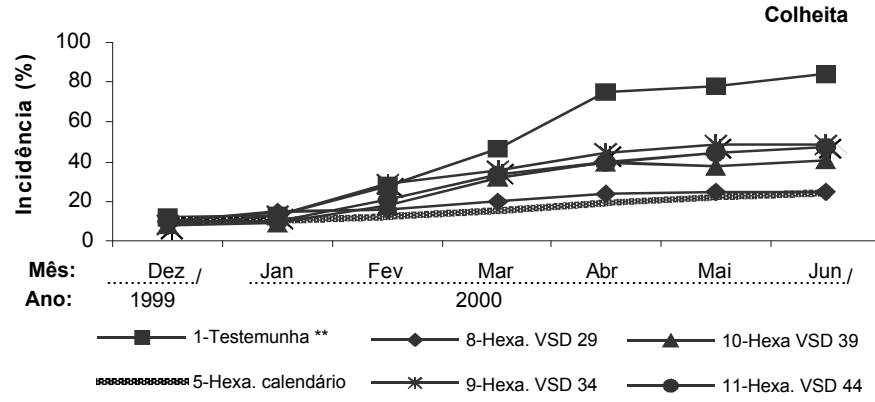

FIG. 1 - Progresso da ferrugem do cafeeiro (Coffea arabica) medido pelo incidência de folhas de café com ferrugem, em lavoura com alta carga pendente de frutos, no ano agrícola 1999/00, em CoimbraMG; (A) Tratamentos: 1- Testemunha; 2- Ox. de cobre 500 PM 3,0 $\mathrm{kg} / \mathrm{ha}$ em Dez/99, Jan/00, Fev/00 e Mar/00 (calendário); 3 Epoxiconazole $250 \mathrm{SC}$ 0,6 1/ha em Jan/00 e 0,4 1/ha em Mar/00 (calendário); 4- Tetraconazole 100 CE 0,5 1/ha em Jan/00 e Mar/00

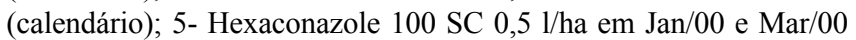
(calendário); 6- Epoxiconazole $250 \mathrm{SC} 0,6$ 1/ha quando atinge $10 \%$ de incidência e 60 dias após primeira 0,4 1/ha; 7- Hexaconazole 100 SC: $0,51 /$ ha quando atingir o índice de $10 \%$ de incidência, e 60 dias após primeira 0,5 1/ha; (B) Tratamentos: 1- Testemunha; 5Hexaconazole 100 SC 0,5 1/ha em Jan/00 e Mar/00 (calendário); 8Hexaconazole $100 \mathrm{SC}$ : 0,5 1/ha quando atingir VSF 29; 9Hexaconazole 100 SC: 0,5 1/ha quando atingir VSF 34; $10-$ Hexaconazole $100 \mathrm{SC}$ : 0,5 1/ha quando atingir VSF $39 ; 11$ Hexaconazole $100 \mathrm{SC} 0,5$ 1/ha quando atingir VSF 44. Os tratamentos 1- Testemunha e 5- Hexaconazole 100 SC (calendário), foram incluídos nas duas figuras para efeito de comparação. que está de acordo com os resultados de Vale et al. (2000). A segunda razão, é a redução de formação de folhas no cafeeiro, a partir de março (Barros \& Maestri, 1974), devido à diminuição do fotoperíodo. De acordo com Nutman \& Roberts (1963), a germinação de uredosporos é mais freqüente em folhas novas do que em folhas velhas, e mais acentuada nas margens do que próxima à nervura principal. Mahlow \& Moraes (1983), também verificaram a influência da idade da folha de café no processo infetivo da ferrugem, concluindo que o primeiro e o segundo par de folhas são mais suscetíveis à infecção por $H$. vastatrix, provavelmente devido a alterações quantitativas e qualitativas de compostos fenólicos nas folhas de maior idade, logo estes fatos limitam novas infecções a partir de abril.

Em Coimbra, ocorreu predominância de molhamento foliar com temperaturas médias na faixa $16^{\circ} \mathrm{C}$ a $18^{\circ} \mathrm{C}$, seguida de $19^{\circ} \mathrm{C}$ a $20^{\circ} \mathrm{C}$, sendo respectivamente, de 642 e $300 \mathrm{~h}$, o que corresponde a $48,42 \%$ e $22,62 \%$ do período de molhamento foliar total ocorrido neste ensaio. Sendo que a partir de dezembro de 1999 até março de 2000, houve 903 h de molhamento foliar, o que representa a $68,10 \%$ do total; e em $83,83 \%$ deste período houve temperatura favorável ao desenvolvimento do patógeno. Já a partir de abril, mesmo ocorrendo $31,90 \%$ do período de molhamento, apenas $44,68 \%$ deste apresentou temperatura favorável ao desenvolvimento do patógeno. Logo, se pode concluir que as condições de temperatura, após o mês de abril, limitaram a ocorrência de novas infeções; portanto, as lesões de ferrugem visualizadas após essa data são decorrentes de infeções ocorridas em fevereiro e março.

O teste de agrupamento Scott-Knott permitiu separar quatro grupos de tratamentos distintos quanto à AACPD com base na incidência de folhas de café com ferrugem (Tabela 2A).

Nas condições do ensaio, o método de controle da ferrugem do cafeeiro por meio da incidência de folhas doentes, com índice de $10 \%$ para o início das pulverizações, o tratamento 6 (Epoxiconazole 250 SC quando atinge $10 \%$ de incidência) foi o que apresentou menor AACPF, demonstrando que princípio ativo Epoxiconazole $250 \mathrm{SC}$ apresentou melhor eficiência em relação ao Hexaconazole $100 \mathrm{SC}$, no controle curativo de $H$. vastatrix.

Este resultado está de acordo com Matiello et al. (1995), os quais realizando ensaios de comparação de princípios ativos para o controle de $H$. vastatrix, encontraram que em Rio Preto - RJ, a incidência de folhas de café com ferrugem, por ocasião da colheita em julho de 1995 , foi de $32,5 \%, 6,5 \%$ e $91,4 \%$ para os fungicidas Hexaconazole $100 \mathrm{SC}$, Epoxiconazole $250 \mathrm{SC}$ e a testemunha, respectivamente. Em Eloy Mendes - MG, as incidências da doença considerando os mesmos tratamentos, foram $9,5 \%, 8,0 \%$ e 77,0\%, sendo a diferença entre Epoxiconazole e Hexaconazole, não significativa ao nível de $5 \%$ de probabilidade. Compreendem-se esses resultados quando se analisa as datas de aplicações dos fungicidas. No primeiro ensaio, as aplicações foram realizadas em 20/02/1995 e 22/04/1995, e no segundo, em 05/01/1995 e 10/03/1995; os autores concluíram que devido ao fato de no primeiro ensaio 
Controle da ferrugem do cafeeiro com base no valor...

ter sido aplicado os fungicidas mais tardiamente, o Epoxiconazole 250 SC foi superior no controle da ferrugem em relação ao Hexaconazole 100 SC. Quando aplicados mais cedo, ambos os fungicidas apresentaram controle semelhante da doença.

Como o tratamento 8 (Hexaconazole $100 \mathrm{SC}$ aplicado baseando-se no VSF 29) apresentou resultado semelhante ao tratamento 5 (Hexaconazole $100 \mathrm{SC}$ calendário), baseando-se na AACPF, indica que para anos de alta carga pendente de frutos, o método de controle químico por meio do VSF requer valor em torno de 30 .

\section{Controle baseado no valor de SFC em lavoura com média carga pendente de frutos}

As epidemias de ferrugem em Carmo do Paranaíba iniciaram em janeiro e alcançaram os valores máximos em junho de 2000 (Figura 2), chegando a 33,0\% de incidência no tratamento testemunha.

O teste de agrupamento Scott-Knott, permitiu separar dois grupos de tratamentos distintos quanto a AACPD, com base na incidência de folhas de café com ferrugem (Tabela 2B).

Nota-se que para a lavoura com média carga pendente de frutos, não houve diferença entre os efeitos dos tratamentos baseados no calendário de aplicação de fungicidas, tanto protetor como sistêmico, e os efeitos dos tratamentos baseados no VSF. Nestas condições o VSF como critério para o controle químico da ferrugem previu apenas uma pulverização de fungicida sistêmico, enquanto o calendário recomendou duas pulverizações de fungicida sistêmico.
Como os resultados em eficiência no controle da ferrugem do tratamento 8 (Hexaconazole 100 SC baseado no VSF igual a 49) apresentou resultado semelhante ao do tratamento 5 (Hexaconazole 100 SC calendário), em relação a AACPF e a menor incidência final de folhas com ferrugem na colheita $(11,0 \%)$ dos tratamentos baseados no critério do VSF conclui-se que, para anos de média carga pendente de frutos, deve-se adotar VSF em torno de 49. Entretanto, não foi possível tomar a decisão de aplicar o fungicida apenas considerando o VSF; houve a necessidade de se utilizar os dados de incidência da ferrugem, devido ao fato dos resultados dos tratamentos 9 (VSF 59), 10 (VSF 969) e 11 (VSF 79), não diferiram do tratamento 5, pelo teste de Scott-Knot, em relação a AACPF.

\section{Epidemiologia comparativa da incidência da ferrugem do cafeeiro}

Para o município de Coimbra o molhamento foliar apresentou um total de horas de duração entre a última semana de dezembro de 1999 e última semana de junho de 2000, de 1326 h, já no município de Carmo do Paranaíba, foi de 1378 h, sendo bastante próximo os valores nos dois locais. Porém a temperatura, durante este período de molhamento, foi mais favorável ao desenvolvimento da ferrugem em Carmo do Paranaíba $\left(18,4^{\circ} \mathrm{C}\right)$ do que em Coimbra $\left(17,0^{\circ} \mathrm{C}\right)$.

Quantificando a favorabilidade destas duas variáveis meteorológicas por meio da matriz (Tabela 1), verifica-se que os valores de severidade da doença totais acumuladas foram 71 VSF e 91 VSF, respectivamente, para Coimbra e Carmo do Paranaíba. Desconsiderando os outros fatores que influenciam a epidemia de $H$. vastatrix, a expectativa era uma incidência de

TABELA 2 - Área abaixo da curva de progresso da ferrugem (AACPF), com base na incidência de ferrugem do cafeeiro (Coffea arabica) 1/ha; para os municípios de Coimbra (A) e Carmo do Paranaíba (B)

\begin{tabular}{|c|c|c|c|c|c|}
\hline \multicolumn{3}{|c|}{$\mathbf{A}$} & \multicolumn{3}{|c|}{ B } \\
\hline Tratamento' & $\begin{array}{l}\text { AACPF } \\
\text { (Média) }\end{array}$ & $\begin{array}{c}\text { Incidência } \\
\text { (Junho 2000) }\end{array}$ & Tratamento $^{2}$ & $\begin{array}{l}\text { AACPF } \\
\text { (Média) }\end{array}$ & $\begin{array}{c}\text { Incidência } \\
\text { (Junho 2000) }\end{array}$ \\
\hline 3 & $2813,75 \mathbf{a} *$ & 22,00 & 2 & $186,50 \mathbf{a} *$ & 3,50 \\
\hline 5 & $3023,88 \mathbf{a}$ & 25,00 & 3 & $267,75 \mathbf{a}$ & 4,75 \\
\hline 6 & $3084,00 \mathbf{a}$ & 27,00 & 5 & $401,25 \mathbf{a}$ & 6,25 \\
\hline 2 & $3477,50 \mathbf{a}$ & 28,50 & 8 & $486,63 \mathbf{a}$ & 11,00 \\
\hline 8 & $3528,50 \mathbf{a}$ & 24,50 & 4 & $510,25 \mathbf{a}$ & 8,00 \\
\hline 4 & $4167,50 \mathbf{b}$ & 38,50 & 9 & $715,75 \mathbf{a}$ & 15,00 \\
\hline 7 & $4251,25 \mathbf{b}$ & 38,00 & 11 & $777,75 \mathbf{a}$ & 18,75 \\
\hline 10 & 4872,25 b & 41,00 & 10 & $858,13 \mathbf{a}$ & 19,75 \\
\hline 11 & $5402,38 \mathbf{b}$ & 48,00 & 1 & $1679,63 \mathbf{b}$ & 33,00 \\
\hline 9 & $6042,75 \mathbf{c}$ & 48,50 & 6 & $1911,63 \mathbf{b}$ & 26,25 \\
\hline 1 & $8807,50 \mathbf{d}$ & 84,50 & 7 & $1920,88 \mathbf{b}$ & 31,75 \\
\hline
\end{tabular}

* Médias seguidas de mesmas letras não se diferenciam entre si pelo teste Scott-Knott, ao nível de $5 \%$ de probabilidade ${ }^{1}$ Tratamentos: 1-Testemunha; 2- Ox. de cobre 500 PM 3,0 kg/ha em Dez/99, Jan/00, Fev/00 e Mar/00 (calendário); 3-

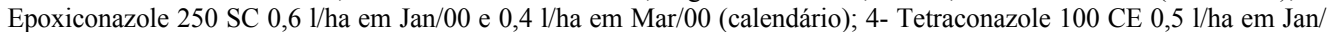

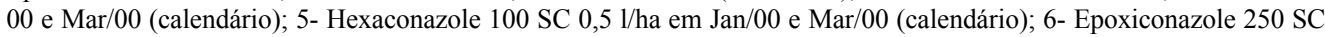
$0,61 /$ ha quando atinge $10 \%$ de incidência e 60 dias após primeira $0,41 /$ ha; 7 - Hexaconazole $100 \mathrm{SC}: 0,5$ 1/ha quando atingir o índice de $10 \%$ de incidência, e 60 dias após primeira 0,5 1/ha; 8- Hexaconazole 100 SC quando VSF atingir 29; 9- Hexaconazole 100 SC quando VSF atingir 34; 10- Hexaconazole 100 SC quando VSF atingir 39 e 11- Hexaconazole 100 SC quando VSF atingir 44; ${ }^{2}$ Tratamentos: 1 a 7 idem (A). 8- Hexaconazole 100 SC: 0,5 1/ha quando atingir VSF 49; 9- Hexaconazole 100 SC: 0,5 1/ha quando atingir VSF 59; 10- Hexaconazole 100 SC: 0,5 l/ha quando atingir VSF 69; 11- Hexaconazole 100 SC 0,5 1/ha quando atingir VSF 79. 


\section{C.L.P. Garçon et al.}

doença ligeiramente superior para a lavoura localizada em Carmo do Paranaíba. Porém, a incidência final de folhas com ferrugem na testemunha, por ocasião da colheita, em Coimbra foi de 84,5\% e em Carmo do Paranaíba de 33,0\%. Esta diferença é explicada pela produção das duas lavouras escolhidas, sendo 101,5 sacas beneficiadas/ha para a primeira localidade, e 22,3 sacas beneficiadas/ha na segunda, o que está de acordo com Miguel et al. (1977), Mansk \& Matiello (1984) e Zambolim et al. (1992).

Para os dois ensaios, tanto o localizado em Coimbra como em Carmo do Paranaíba, as condições de molhamento foliar com temperatura favorável a ocorrência de novas infecções de $H$. vastatrix em condições de campo estenderamse até o mês de março de 2000; porém, o pico de incidência de folhas de café com ferrugem em Coimbra ocorreu no mês de
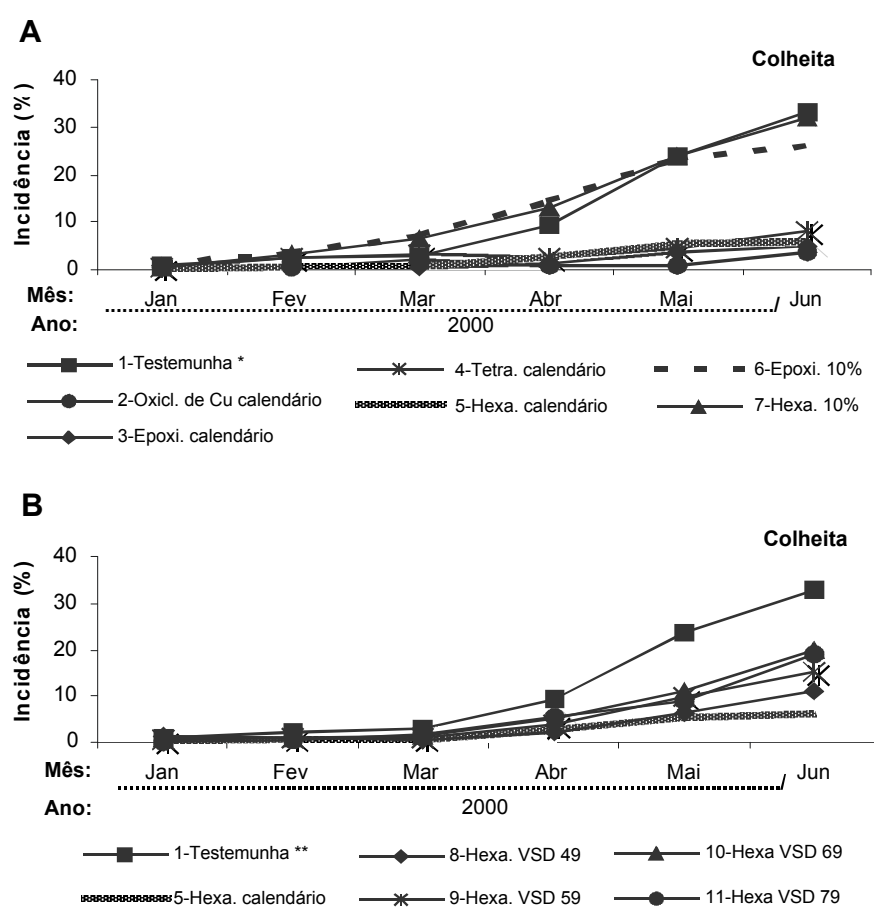

FIG. 2 - Progresso da ferrugem do cafeeiro (Coffea arabica), medido pela incidência de folhas de café com ferrugem, em lavoura com média carga pendente de frutos, no ano agrícola 1999/00, em Carmo do Paranaíba - MG. (A) 1- Testemunha; 2- Ox. de cobre 500 PM 3,0 $\mathrm{kg} / \mathrm{ha}$ em Dez/99, Jan/00, Fev/00 e Mar/00 (calendário); 3 Epoxiconazole $250 \mathrm{SC} 0,6$ 1/ha em Jan/00 e 0,4 1/ha em Mar/00 (calendário); 4- Tetraconazole 100 CE 0,5 1/ha em Jan/00 e Mar/00

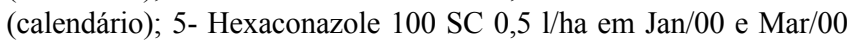
(calendário); 6- Epoxiconazole $250 \mathrm{SC} 0,6$ 1/ha quando atingir $10 \%$ de incidência e 60 dias após primeira 0,4 1/ha; 7- Hexaconazole 100 SC: 0,51 /ha quando atingir o índice de $10 \%$ de incidência, e 60 dias após primeira 0,5 1/ha; (B) 1- Testemunha; 5-Hexaconazole $100 \mathrm{SC}$ 0,5 1/ha em Jan/00 e Mar/00 (calendário); 8- Hexaconazole $100 \mathrm{SC}$ : 0,5 1/ha quando atingir VSF 49; 9- Hexaconazole $100 \mathrm{SC}$ : 0,5 1/ha quando atingir VSF 59; 10- Hexaconazole $100 \mathrm{SC}$ : 0,5 1/ha quando atingir VSF 69; 11- Hexaconazole $100 \mathrm{SC} 0,5$ 1/ha quando atingir VSF 79. Os tratamentos 1- Testemunha e 5- Hexaconazole 100 SC (calendário), foram incluídos nas duas figuras para efeito de comparação. abril de 2000, 30 dias após o término das condições ambientais favoráveis a novas infecções de $H$. vastatrix. Um mês após o término das condições favoráveis para que ocorresse infecção, os sintomas já eram visíveis, o que indica um período latente curto (inferior a 30 dias). Em Carmo do Paranaíba, o pico da doença ocorreu em junho de 2000, em torno de 90 dias após o término das condições ambientais favoráveis a novas infecções de $H$. vastatrix. Houve um atraso de três meses para visualização dos sintomas da maioria das infecções de ferrugem nessa localidade, o que indica um período latente mais longo do que o ocorrido em Coimbra,o que pode ser explicado pela altitude da lavoura de café onde os experimentos foram instalados; em Carmo do Paranaíba, a altitude do cafezal onde o experimento foi instalado, $850 \mathrm{~m}$, é maior do que em Coimbra $680 \mathrm{~m}$.

Os resultados comprovam que normalmente, em lavouras com baixa a média carga pendente de frutos, uma única aplicação de fungicida sistêmico no momento oportuno, o qual é indicado com base no número de horas de molhamento foliar e na temperatura média durante o período de molhamento, associado à carga pendente de frutos, como sugerido pelo sistema de VSF do cafeeiro identificado, foi suficiente para se alcançar controle eficiente da ferrugem do cafeeiro, com maior economia para o agricultor e menor agressão ao ambiente.

\section{REFERÊNCIAS BIBLIOGRÁFICAS}

ALFONSI, R.R., ORTOLANI, A.A., PINTO, H.S., PEDRO JR., M.J. \& BRUNINI, O. Associação entre nível de infecção da ferrugem do cafeeiro, variáveis meteorológicas e área foliar, observados em (Coffea arabica L). Resumos, 2 Congresso Brasileiro de Pesquisas Cafeeiras, Poços de Caldas, MG. 1974. pp.80-83.

BARROS, R.S. \& MAESTRI, M. Influência dos fatores climáticos sobre a periodicidade de crescimento vegetativo do café (Coffea arabica L.). Revista Ceres 21:268-279. 1974.

CAMPBELL, C.L. \& MADDEN, L.V. Introduction to Plant Disease Epidemiology. New York. John Wiley \& Sons Inc. 1990.

GREE, G. Epidemiology of coffee leaf rust in the Eastern Highlands. Newsletter - Coffee - Research - Institute 2:16-20. 1993.

KUSHALAPPA, A.C., AKUTSU, M. \& LUDWIG, A. Application of survival ratio monocyclic process of Hemileia vastatrix in predicting coffee rust infection rates. Phytopathology 73:96-103. 1983.

MAFFIA, L.A., SILVA-ACUÑA, R., ZAMBOLIM, L. \& BERGER, D. Incidence-severity relationships on the pathosystem Coffea arabica L. - Hemileia vastatrix Berk. et Br. Plant Disease 83:186188. 1999.

MAHLOW, M.F.P. \& MORAES, W.B.C. Dinâmica de infecção de folhas de café por Hemileia vastatrix. Resumos, $10^{\circ}$ Congresso Brasileiro de Pesquisas Cafeeiras, Poços de Caldas, MG. 1983. p.102.

MANSK, Z. \& MATIELLO, J.B. Efeito da produção, nível de desfolha e inóculo residual sobre a evolução da ferrugem do cafeeiro no Estado do Espírito Santo. Resumos, 11ํㅡㄹ Congresso Brasileiro de Pesquisas Cafeeiras, Londrina, PR. 1984. pp.41-43.

MATIELLO, J.B., ALMEIDA, S.R. \& FEREIRA, R.A. Eficiência do hexaconazole (Anvil) em associação com zinco e cobre no controle 
Controle da ferrugem do cafeeiro com base no valor...

da ferrugem do cafeeiro. Resumos, 21ํ Congresso Brasileiro de Pesquisas Cafeeiras, Caxambu, MG. 1995. pp.35-37.

MIGUEL, A.E., MATIELlO, J.B., MANSK, Z. \& ALMEIDA, S.R. Observações sobre os efeitos de três níveis de produção na incidência e controle da ferrugem do cafeeiro. Resumos, $5^{\circ}$ Congresso Brasileiro de Pesquisas Cafeeiras, Guarapari, ES. 1977. pp.220-221.

NUTMAN, F.J. \& ROBERTS, F.M. Studies on the biology of Hemileia vastatrix Berk \& Br. Transactions British Mycological Society 46:27-48. 1963.

ROTEM, J. The Genus Alternaria. Biology, Epidemiology and Pathogenicity. St. Paul, Minnesota. APS PRESS. 1994.

VALE, F.X.R., ZAMBOLIM, L. \& JESUS Jr., W.C. Efeito de fatores climáticos na ocorrência e no desenvolvimento da ferrugem do cafeeiro. Resumos, I Simpósio de Pesquisa dos Cafés do Brasil, Poços de Caldas, MG. 2000. pp.171-174.

WALLIN, J. R. Summary of recent progress in predicting late blight epidemics in United States and Canada. American Potato Journal
39:306-312. 1962.

ZAMBOLIM, L., ACUÑA, R.S., VALE, F.X.R. \& CHAVES, G.M. Influência da produção do cafeeiro sobre o desenvolvimento da ferrugem (Hemileia vastatrix Berk. et Br.). Fitopatologia Brasileira 17:32-35. 1992.

ZAMBOLIM, L., VALE, F.X.R., PEREIRA, A.A. \& CHAVES, G.M. Café (Coffea arabica L.), controle de doenças causadas por fungos, bactérias e vírus. In: Vale, F.X.R. \& Zambolim, L. (Eds.) Controle de doenças de plantas. Viçosa, Minas Gerais. Suprema Gráfica e Editora. 1997. pp.83-180.

ZAMBOLIM, L. \& VALE, F.X.R. Princípios de Fitopatologia. ABEAS - Curso de Proteção de Plantas - módulo 5. Brasília. 1999.

ZAMBOLIM, L., VALE, F.X.R., COSTA, H., PEREIRA, A.A. \& CHAVES, G.M. Epidemiologia e controle da ferrugem do cafeeiro (Hemileia vastatrix Berk. et Br.). In: Zambolim, L. (Ed.) O Estado da Arte de Tecnologias na Produção de Café. Viçosa, Minas Gerais. Suprema Gráfica e Editora. 2002. pp.369-433. 